\title{
The Impact of Chronic Urticaria from the Patient's Perspective: A Survey in Five European Countries
}

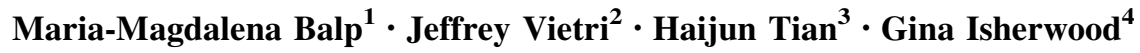

Published online: 17 October 2015

(C) The Author(s) 2015. This article is published with open access at Springerlink.com

\begin{abstract}
Background Chronic spontaneous urticaria (CSU) is associated with considerable burden, but data from European patients are limited.

Methods This study is a retrospective, cross-sectional analysis of National Health and Wellness Survey data from the five largest EU countries (5EU: France, Germany, Italy, Spain, and the UK) collected between 2010 and 2013. Burden of disease for patients with CSU was estimated by comparing individuals currently treated for chronic urticaria (proxy CSU cases) with controls selected from respondents without chronic urticaria. Matching and regression models were used to quantify the impact of chronic urticaria on health-related quality of life, self-reported psychological complaints, work and activity impairment, and healthcare use.

Results The sample included 175,923 respondents. Prevalence of diagnosed chronic urticaria was 0.5 and $0.2 \%$ were treating the condition with a prescription. Cases $(N=369)$ had substantially lower (worse) regression-adjusted mean Mental Component Summary (40.2 vs.
\end{abstract}

Electronic supplementary material The online version of this article (doi:10.1007/s40271-015-0145-9) contains supplementary material, which is available to authorized users.

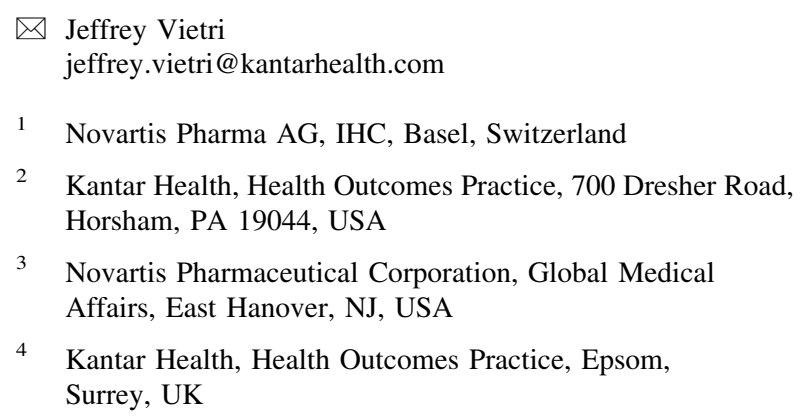

4 Kantar Health, Health Outcomes Practice, Epsom, Surrey, UK

45.4), Physical Component Summary (44.6 vs. 49.9), and SF-6D health utility scores $(0.63$ vs. 0.71 ; all $p<0.001)$ relative to controls $(N=1476)$, differences that exceed accepted minimally important differences for these measures. Depression, anxiety, and sleep difficulties were approximately twice as prevalent among those currently receiving treatment for chronic urticaria (all $p<0.001$ ). Cases also had elevated presenteeism (31 vs. $17 \%$ ), overall work impairment ( 37 vs. $20 \%$ ), and impairment in non-work activities ( 42 vs. $26 \%$; all $p<0.01$ ) relative to controls. Physician visits (9.1 vs. 4.9), emergency room visits $(0.8$ vs. 0.3$)$, and hospitalizations ( 0.3 vs. 0.2$)$ were more frequent than in controls (all $p<0.01$ ).

Conclusions This research adds to the existing evidence showing significant burden of CSU.

\section{Key Points for Decision Makers}

One in every 200 adults in the five largest countries in the EU has been diagnosed with chronic urticaria, and $40 \%$ of them are currently using a prescription treatment.

The impact on patients' lives goes beyond effects on skin; currently treated patients experience higher levels of health-related impairment in their functioning in work and non-work activities and quality of life, and are more frequent users of healthcare than similar individuals without the condition.

Practitioners need to be aware of the treatment options and find something that works for each patient. 


\section{Introduction}

Chronic spontaneous urticaria (CSU) is defined as the "spontaneous appearance of wheals, angioedema or both $\geq 6$ weeks due to known and unknown causes" [1]. CSU often has no discernable external cause and is associated with an underlying autoimmune mechanism, which can be confirmed by testing for autoantibodies, in 30-50\% of cases [2,3]. CSU is the most common type of chronic urticaria, accounting for approximately two-thirds of all cases [4]. Only a few studies have examined the prevalence of CSU in European countries, and these estimate the rate at between 0.1 and $0.8 \%$ in samples from Sweden, Spain, and Germany [5-7]. The disease is most common among patients aged 20-40 years, but all age groups can be affected [4]. Studies also consistently show that women suffer from this disease at almost twice the rate of men [6-8].

The burden of CSU can be considerable. Published data on patients with chronic urticaria, including studies specific to CSU patients as well as studies examining chronic urticaria without differentiating between types, indicate that issues with fatigue, pain, and lack of sleep or insomnia are commonly reported, often due to the constant itching that accompanies their urticaria [9]. The visible lesions can lead to emotional upset and withdrawal from social activities [10, 11]. Indeed, CSU can lead to psychological complaints such as anxiety, depression, or irritability [9], which can result in significant reductions in quality of life among CSU patients [12]. In addition to physical and emotional outcomes, some data indicate CSU symptoms can carry a high socioeconomic burden through a combination of direct healthcare costs and loss of work productivity [7].

While there is some literature on the relationship between CSU and economic and quality-of-life outcomes, research in this area remains limited, including few studies conducted among Europeans with the condition. Previous research characterizing the condition has drawn samples primarily from individual clinics or small geographic areas; to the authors' knowledge, no study has been conducted describing the humanistic and economic burden within a general population survey [5-12]. The current study estimates the burden of CSU among adults diagnosed with chronic urticaria in terms of health-related quality of life (HR-QOL), self-reported depression, anxiety, and sleep difficulties, work and activity impairment, and healthcare use, using individuals currently using prescription treatment for chronic urticaria as a proxy for CSU.

\section{Methods}

\subsection{Study Design}

The present study is a retrospective, cross-sectional analysis of data taken from the 5EU National Health and Wellness Survey (NHWS), which is a large cross-sectional survey designed to reflect health in the general population of France, Germany, Italy, Spain, and the UK. Potential respondents are identified primarily through participation in opt-in online survey panels, with stratified random sampling within the survey panel to ensure representativeness in terms of age and sex, and off-panel recruitment is used to ensure representation of the elderly population using quotas by age and sex. Previous comparisons have shown NHWS data to be consistent with that of other health surveys meant to represent the general population $[13,14]$. The current project incorporates data from the 5EU surveys conducted in $2010(N=57,805)$, $2011(N=57,512)$, and $2013(N=62,000)$; the 5EU NHWS was not conducted in 2012. Because the survey is cross-sectional, and sampling is conducted without regard to previous participation, some respondents have completed the survey more than once. The most recent responses were used for such respondents. Respondents were required to be aged at least 18 years, able to read and write the primary language of the country in which the study was conducted, and to provide informed consent. The protocol and questionnaire for the NHWS was reviewed and approved by Essex Institutional Review Board (Lebanon, New Jersey, USA).

\subsection{Measures}

\subsubsection{Chronic Spontaneous Urticaria Proxy}

Items to identify respondents with CSU in particular were not included in the NHWS, but the survey respondents were asked to identify which medical conditions they had ever experienced from a list that included "chronic hives (lasting 6 weeks or more)". The term "hives" was used rather than "urticaria" to ensure the language would be understood by patients rather than health professionals. No description of the condition was provided. Those who indicated experiencing chronic hives were asked to indicate whether their chronic hives were diagnosed by a doctor, and whether they were currently using a prescription to treat their chronic hives. The type of prescription was not collected. Those who indicated current use of a prescription were used as the proxy for individuals with CSU (cases) to ensure both diagnosis of chronic urticaria as well as present disease at the time of the survey. 


\subsubsection{Health-Related Quality of Life}

The NHWS assessed HR-QOL using the standard recall form (4 weeks) of the SF-12v2 for 2010 and 2011 and the SF-36v2 for the 2013 responses $[15,16]$. These are both generic (i.e., not disease specific) HR-QOL instruments that allow for comparison across health conditions and can be used to produce the same metrics for functional health status. Mental Component Summary (MCS) scores were used to summarize mental health, and Physical Component Summary (PCS) scores were used to summarize physical health. MCS and PCS scores have a mean of 50 and a standard deviation of 10 for the population. Health utility scores were also calculated using the SF-6D algorithm, which provides a preference-based single index measure for health using UK general population values, with a score of 1 equivalent to full health [17].

\subsubsection{Self-Report of Depression, Anxiety, and Sleep Problems}

Respondents to the survey were asked to indicate which medical conditions they had experienced in the prior 12 months, one of which was depression. Those who indicated experience of depression were considered to have depression. Respondents to the survey were also asked to indicate whether they have experienced a variety of different types of anxiety in the preceding 12 months, including anxiety, general anxiety disorder, panic disorder, phobia, post-traumatic stress, obsessive compulsive disorder, and social anxiety disorder. If a respondent indicated having experienced one or more of these in the past 12 months, they were considered to have anxiety. Likewise, respondents who indicated they had experienced insomnia or sleep difficulties in the prior 12 months were considered to have sleep difficulties. For all conditions, the name of the condition was presented to the respondent without additional description.

\subsubsection{Work and Activity Impairment}

The Work Productivity and Activity Impairment questionnaire was included in the study to measure health-related work and activity impairment [18]. Only respondents who reported being employed full-time, part-time, or selfemployed provided data for absenteeism (work time missed due to health), presenteeism (level of health-related impairment while at work), and overall work impairment (combined absenteeism and presenteeism). All respondents provided data for activity impairment (health-related impairment to non-work activities). All values are reported as percentages and are based on the 7 days prior to the survey.

\subsubsection{Healthcare Utilization}

Healthcare use was defined by visits to different medical providers during the prior 6 months. These included visits to specific types of traditional healthcare providers (HCPs: physicians, nurses, dentists, etc.), which were summed across types of providers to provide a total. Specific types of providers who would be expected to provide care for CSU or the common psychological comorbidities were also considered individually, including general practitioners (GPs), allergists, dermatologists, psychiatrists, and psychologists. Respondents also provided the number of emergency room visits and hospitalizations. Whether the respondent made at least one visit to various types of alternative HCPs (herbalists, acupuncturists, chiropractors, nutritionists, massage therapists, and homeopaths) was also included in the survey, though the number of such visits was not asked.

\subsubsection{Demographic and Clinical Characteristics}

A series of demographic and clinical characteristics were also included to characterize the sample and serve as covariates in the regression models. Age, sex, country of residence, marital status, level of household income, and employment status were included as demographic measures. General health characteristics included body mass index (BMI) calculated from reported height and weight, cigarette smoking, frequency of alcohol use, and days of exercise in past month. The Charlson comorbidity index (CCI) was also calculated from self-reported physician diagnoses of different conditions to represent the level of comorbidity among respondents [19]. The greater the total index score, the greater the comorbid burden on the patient.

\subsection{Analysis}

Point prevalence of CSU among adults in the 5EU was estimated using the three possible definitions available in the NHWS (ever experienced, diagnosed by a physician, and currently using a prescription) by applying HorvitzThompson weights to respondents according to country, sex, and age as reported for each country in the international database of the US Census in 2013 [20, 21].

In order to quantify burden, cases were matched to $5 \mathrm{EU}$ NHWS respondents who had not experienced chronic urticaria (controls) in a 1:4 ratio. The matching was random without replacement among those sharing the same country, sex, age (exact), and year of survey. Comparisons between cases and matched controls were conducted using chi-squared tests of categorical variables and $t$-tests for continuous and count variables. Generalized linear models were also conducted to adjust for potential confounders. 
Table 1 Prevalence of chronic urticaria in the 5EU (France, Germany, Italy, Spain, and UK)

\begin{tabular}{llll}
\hline Chronic urticaria & Overall & Male & Female \\
\hline Ever experienced & $0.63(0.59-0.67)$ & $0.46(0.41-0.51)$ & $0.79(0.72-0.85)$ \\
Ever diagnosed & $0.51(0.48-0.55)$ & $0.34(0.30-0.38)$ & $0.67(0.62-0.73)$ \\
Currently treated & $0.23(0.20-0.25)$ & $0.18(0.14-0.21)$ & $0.28(0.24-0.31)$ \\
\hline
\end{tabular}

Data are presented as \% (95\% confidence interval)

Currently treated respondents are a subset of ever-diagnosed respondents, which is a subset of those who ever experienced chronic urticaria
These models took into account cigarette smoking, BMI category, and CCI score, which differed between cases and controls after matching. Models were selected based on the distribution of the outcomes. As the distribution of HRQOL metrics was roughly normal, linear models were used for these outcomes. Logistic regression was used for depression, anxiety, and sleep difficulties, as these were binary outcomes. Negative binomial models with a log link function were used for all other outcomes, as the distributions were positively skewed and also were over-dispersed for the Poisson distribution. ${ }^{1}$ To ease interpretation of the regression models, regression-estimated means are presented at the mean value of the covariates. Additional detail for the regression results, such as significance tests and coefficients for the covariates, odds ratios, and rate ratios, are presented in the Electronic Supplementary Material (ESM) 1.

\section{Results}

There were 175,923 unique respondents across the 3 years of survey data. The prevalence of chronic urticaria currently treated with a prescription was estimated at $0.23 \%$ of the adult population after weighting, with an additional $0.28 \%$ reporting a physician diagnosis, for a prevalence of $0.51 \%$ for diagnosed chronic urticaria. A further $0.12 \%$ reported having experienced chronic urticaria but not having received a diagnosis, for a total prevalence of $0.63 \%$ when considering both diagnosed and undiagnosed cases. The condition was almost twice as common among women as among men, regardless of definition used (Table 1).

Bivariate comparisons between cases $(n=369)$ and matched controls $(n=1476)$ indicated that those currently treated for chronic urticaria (cases) were not significantly different from controls in terms of household income, marital status, level of education, frequency of exercise, or

\footnotetext{
${ }^{1}$ A series of generalized linear mixed models accounting for the matched structure of the data was also conducted, and resulted in similar conclusions; these results are available from the corresponding author. Because of the similarity in findings and an issue with non-convergence for a few outcomes, only the results of the generalized linear models are presented here.
}

alcohol use (all $p>0.10$ ). Cases were less likely to be employed (51.5 vs. $58.6 \%, p<0.05)$, had higher mean BMI (26.6 vs. $25.5, p<0.01)$, and differed in the distribution of BMI and smoking categories (both $p<0.01$ ), which was due to a higher proportion of cases being obese (23.0 vs. $15.9 \%)$ and currently smoking cigarettes (35.5 vs. $28.2 \%$ ) than controls. CCI scores were notably higher among those treated for chronic urticaria than among the controls (1.7 vs. 0.3, $p<0.001$; Table 2 ).

All health outcomes were considerably worse among cases than among matched control respondents. HR-QOL was significantly worse among those currently treated for chronic urticaria than among those who had never experienced chronic urticaria (Fig. 1a), including mean MCS scores (39.6 vs. 45.5 ), PCS scores (43.4 vs. 50.2), and SF$6 \mathrm{D}$ health utility scores $(0.62$ vs. 0.71 ; all $p<0.001)$. Substantial decrements in HR-QOL outcomes remained among those currently treated for chronic urticaria after adjusting for BMI category, CCI, and smoking, with the adjusted mean scores for cases approximately 5 points lower on both MCS (40.2 vs. $45.4, p<0.001)$ and PCS (44.6 vs. 49.9$)$ scores $(p<0.001)$. Health utility scores were 0.08 points lower after adjustment $(0.63$ vs. 0.71 , $p<0.001$ ) (Fig. 1b).

There was also an association between current treatment for chronic urticaria and psychological complaints (Table 3). Self-reported depression was approximately twice as prevalent among cases than among controls (32.8 vs. $14.6 \%, p<0.001)$. Anxiety was experienced by approximately half of cases, again approximately double the rate among controls (50.9 vs. $22.6 \%, p<0.001)$, and sleep difficulties were also more common in this group than among controls (58.0 vs. $32.7 \%, p<0.001)$. The difference in prevalence of these complaints after adjustment for confounders, was similar to the unadjusted figures. The estimated probability of depression was 27.5 vs. $14.7 \%$ for cases and controls, respectively, and the prevalence of anxiety was $47.0 \%$ for cases and $23.3 \%$ for controls (both $p<0.001$ ). The adjusted prevalence of sleep difficulties was also higher among cases than controls (54 vs. $34 \%, p<0.001$; Table 3 ).

Impairment in work and activities is summarized in Table 4. Average levels of absenteeism (13.4 vs. $5.6 \%$, $p<0.001$ ), presenteeism (33.5 vs. $16.6 \%, p<0.0001$ ), 
Table 2 Patient characteristics in respondents currently treated for chronic urticaria and matched controls

\begin{tabular}{|c|c|c|c|}
\hline Characteristic & $\begin{array}{l}\text { Control } \\
(N=1476)\end{array}$ & $\begin{array}{l}\text { Currently treated for } \\
\text { chronic urticaria } \\
(N=369)\end{array}$ & $p$ value \\
\hline Age, mean (SD) & $44.4(15.2)$ & $44.4(15.2)$ & 1.000 \\
\hline Male & $512(34.7)$ & $128(34.7)$ & 1.000 \\
\hline Country & & & 1.000 \\
\hline France & $356(24.1)$ & $89(24.1)$ & \\
\hline Germany & $252(17.1)$ & $63(17.1)$ & \\
\hline UK & $276(18.7)$ & $69(18.7)$ & \\
\hline Italy & $320(21.7)$ & $80(21.7)$ & \\
\hline Spain & $272(18.4)$ & $68(18.4)$ & \\
\hline Employed & $865(58.6)$ & $190(51.5)$ & 0.014 \\
\hline Household income & & & 0.125 \\
\hline Below median & $734(49.7)$ & $202(54.7)$ & \\
\hline Above median & $523(35.4)$ & $125(33.9)$ & \\
\hline Declined to answer & $219(14.8)$ & $42(11.4)$ & \\
\hline Married/living w/partner & $913(61.9)$ & $224(60.7)$ & 0.684 \\
\hline Completed university & $603(40.9)$ & $140(37.9)$ & 0.307 \\
\hline BMI, mean $(\mathrm{SD})(n=1433[362])$ & $25.53(5.15)$ & $26.64(6.60)$ & $0.003 *$ \\
\hline Exercise (days in past month) & $6.37(8.21)$ & $6.11(7.81)$ & 0.572 \\
\hline CCI, mean (SD) & $0.29(0.77)$ & $1.72(5.02)$ & $<0.001 *$ \\
\hline BMI categories & & & 0.009 \\
\hline Underweight & $50(3.4)$ & $16(4.3)$ & \\
\hline Normal & $707(47.9)$ & $152(41.2)$ & \\
\hline Overweight & $442(29.9)$ & $109(29.5)$ & \\
\hline Obese & $234(15.9)$ & $85(23.0)$ & \\
\hline Declined to answer & $43(2.9)$ & $7(1.9)$ & \\
\hline Smoking status & & & 0.009 \\
\hline Current & $416(28.2)$ & $131(35.5)$ & \\
\hline Former & $460(31.2)$ & $115(31.2)$ & \\
\hline Never & $600(40.7)$ & $123(33.3)$ & \\
\hline Alcohol use & & & 0.276 \\
\hline Daily & $101(6.8)$ & $32(8.7)$ & \\
\hline Less than daily & $972(65.9)$ & $248(67.2)$ & \\
\hline None & $403(27.3)$ & 89 (24.1) & \\
\hline
\end{tabular}

Data are presented as $n(\%)$ unless otherwise indicated

$B M I$ body mass index, $C C I$ Charlson comorbidity index, $S D$ standard deviation

$* p$ value adjusted for violation of homogeneity of variance assumption and overall work impairment (39.7 vs. $20.1 \%, p<0.001$ ) among employed cases were almost double those reported by employed matched respondents who had never experienced chronic urticaria. Impairment in non-work activities was also nearly doubled among cases relative to controls (45.5 vs. $25.1 \%, p<0.001$ ). Regression adjustment did little to alter the pattern of results, though the difference in absenteeism was no longer significant (11.9 vs. $5.3 \%$, $p=0.058)$. Adjusted comparisons for presenteeism (30.5 vs. $16.9 \%$ ), overall work impairment (36.6 vs. $20.3 \%$ ), and impairment in non-work activities (42.2 vs. $25.5 \%$ ) all indicated greater impairment among cases than controls (all $p<0.01$ ).

Higher levels of healthcare use among cases were also reported by proxy CSU cases (Table 5). Those currently treated for chronic urticaria had approximately six more total HCP visits on average during the 6-month recall period (11.5 vs. $4.8, p<0.001)$. Analysis of visits to specific healthcare providers revealed that cases made more visits to dermatologists, allergists, psychiatrists, and psychologists (all $p<0.05$ ). Cases also had notably more emergency visits $(1.1 \quad$ vs. $0.3, \quad p<0.001)$ and 
(a)

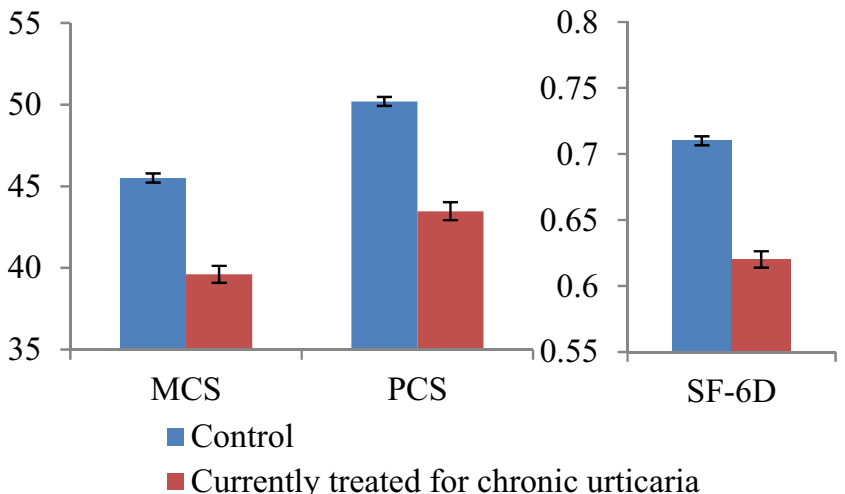

Fig. 1 Health-related quality of life among those currently treated for chronic urticaria relative to controls. $\mathbf{a}$ and $\mathbf{b}$ present matched comparisons before and after regression adjustment, respectively. Variables used in matching were year of survey, country, age, and (b)
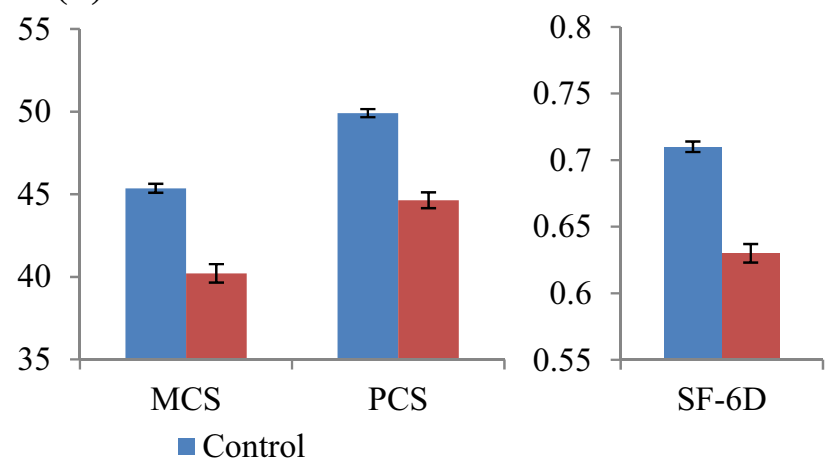

- Currently treated for chronic urticaria

sex. Covariates included cigarette smoking, body mass index category, and Charlson comorbidity index score. Error bars indicate standard error of the mean. All comparisons $p<0.001$. MCS Mental Component Summary, PCS Physical Component Summary

Table 3 Depression, anxiety, and sleep difficulties among respondents currently treated for chronic urticaria and matched controls

\begin{tabular}{|c|c|c|c|c|c|c|}
\hline & \multicolumn{3}{|c|}{ Matched comparisons } & \multicolumn{3}{|c|}{ Regression estimates } \\
\hline & $\begin{array}{l}\text { Control } \\
(N=1476)\end{array}$ & $\begin{array}{l}\text { Currently treated for chronic } \\
\text { urticaria }(N=369)\end{array}$ & $p$ value & $\begin{array}{l}\text { Control } \\
(N=1476)\end{array}$ & $\begin{array}{l}\text { Currently treated for chronic } \\
\text { urticaria }(N=369)\end{array}$ & $p$ value \\
\hline Depression & $216(14.6)$ & $121(32.8)$ & $<0.001$ & $14.7(12.9-16.7)$ & $27.5(23.0-32.5)$ & $<0.001$ \\
\hline Anxiety & $333(22.6)$ & $188(50.9)$ & $<0.001$ & $23.3(21.1-25.6)$ & $47.0(41.7-52.4)$ & $<0.001$ \\
\hline Sleep difficulties & $483(32.7)$ & $214(58.0)$ & $<0.001$ & $34.2(31.7-36.8)$ & $54.3(48.9-59.6)$ & $<0.001$ \\
\hline
\end{tabular}

Data are presented as $n(\%)$ or $\%$ (95\% confidence interval)

Variables used in matching were year of survey, country, age, and sex. Covariates included cigarette smoking, BMI category, and CCI score $B M I$ body mass index, $C C I$ Charlson comorbidity index

Table 4 Impairment in work productivity and non-work activities in respondents currently treated for chronic urticaria and matched controls

\begin{tabular}{|c|c|c|c|c|c|}
\hline \multicolumn{3}{|c|}{ Matched comparisons } & \multicolumn{3}{|c|}{ Regression estimates } \\
\hline $\begin{array}{l}\text { Control } \\
(N=1476)\end{array}$ & $\begin{array}{l}\text { Currently treated for chronic } \\
\text { urticaria }(N=369)\end{array}$ & $p$ value & $\begin{array}{l}\text { Control } \\
(N=1476)\end{array}$ & $\begin{array}{l}\text { Currently treated for chronic } \\
\text { urticaria }(N=369)\end{array}$ & $p$ value \\
\hline
\end{tabular}

\begin{tabular}{lrlllll}
\hline Work impairment & & & & & & \\
Absenteeism & $5.6(18.6)$ & $13.4(26.5)$ & $<0.001$ & $5.3(3.8-7.4)$ & $11.9(5.6-25.3)$ & 0.058 \\
Presenteeism & $16.6(22.8)$ & $33.5(29.9)$ & $<0.001^{*}$ & $16.9(14.5-19.6)$ & $30.5(22.0-42.3)$ & 0.001 \\
Overall & $20.1(27.5)$ & $39.7(33.8)$ & $<0.001$ & $20.3(17.5-23.5)$ & $36.6(26.7-50.2)$ & 0.001 \\
Activity impairment & $25.1(28.1)$ & $45.5(30.8)$ & $<0.001^{*}$ & $25.5(23.3-27.8)$ & $42.2(35.2-50.5)$ & $<0.001$
\end{tabular}

Data are presented as mean \% (standard deviation) or mean \% (95\% confidence interval)

Variables used in matching were year of survey, country, age, and sex. Covariates included cigarette smoking, BMI category, and CCI score $B M I$ body mass index, $C C I$ Charlson comorbidity index, $S D$ standard deviation

* $p$ value adjusted for violation of homogeneity of variance assumption

hospitalizations ( 0.7 vs. $0.2, p<0.001)$ than controls. The pattern of more visits among cases than controls was also seen in the regression results (Table 5). The number of physician visits was approximately twice as high for those currently treated for chronic urticaria relative to controls (9.1 vs. $4.9, p<0.001)$. The pattern was fairly consistent across types of providers, including GPs, allergists, dermatologists, and psychiatrists (all $p<0.05$ ), though psychotherapist visits were not significantly different after adjustment $(p=0.11)$. The disparity between cases and controls in ER visits and hospitalizations was smaller after adjustment, but still significant (both $p<0.01$ ). 
Table 5 Number of all-cause healthcare visits during the prior 6 months among respondents currently treated for chronic urticaria and matched controls

\begin{tabular}{|c|c|c|c|c|c|c|}
\hline \multirow[t]{2}{*}{ Type of provider } & \multicolumn{3}{|c|}{ Matched comparisons } & \multicolumn{3}{|c|}{ Regression estimates } \\
\hline & $\begin{array}{l}\text { Control } \\
(N=1476)\end{array}$ & $\begin{array}{l}\text { Currently treated for chronic } \\
\text { urticaria }(N=369)\end{array}$ & $p$ value & $\begin{array}{l}\text { Control } \\
(N=1476)\end{array}$ & $\begin{array}{l}\text { Currently treated for chronic } \\
\text { urticaria }(N=369)\end{array}$ & $p$ value \\
\hline Physician & $4.82(6.77)$ & $11.47(18.81)$ & $<0.001$ & $4.87(4.61-5.15)$ & $9.06(8.15-10.09)$ & $<0.001$ \\
\hline GP & $1.87(2.54)$ & $3.73(5.05)$ & $<0.001$ & $1.88(1.76-2.00)$ & $3.52(3.13-3.96)$ & $<0.001$ \\
\hline Allergist & $0.04(0.33)$ & $0.34(0.89)$ & $<0.001$ & $0.04(0.03-0.05)$ & $0.30(0.21-0.41)$ & $<0.001$ \\
\hline Dermatologist & $0.11(0.57)$ & $0.56(1.31)$ & $<0.001 *$ & $0.11(0.09-0.13)$ & $0.50(0.38-0.66)$ & $<0.001$ \\
\hline Psychotherapist & $0.17(1.39)$ & $0.42(2.13)$ & $0.033 *$ & $0.11(0.07-0.17)$ & $0.24(0.10-0.56)$ & 0.107 \\
\hline Psychiatrist & $0.16(1.49)$ & $0.41(1.72)$ & $0.010^{*}$ & $0.14(0.09-0.20)$ & $0.39(0.18-0.85)$ & 0.023 \\
\hline ER & $0.30(2.00)$ & $1.12(3.35)$ & $<0.001$ & $0.28(0.24-0.33)$ & $0.75(0.57-0.99)$ & $<0.001$ \\
\hline Hospital & $0.18(1.34)$ & $0.66(2.45)$ & $<0.001 *$ & $0.16(0.13-0.20)$ & $0.33(0.22-0.49)$ & 0.002 \\
\hline
\end{tabular}

Data are presented as mean (standard deviation) or mean (95\% confidence interval)

Variables used in matching were year of survey, country, age, and sex. Covariates included cigarette smoking, BMI category, and CCI score $B M I$ body mass index, $C C I$ Charlson comorbidity index, $E R$ emergency room, $G P$ general practitioner, $R R$ rate ratio

* $p$ value adjusted for violation of homogeneity of variance assumption

\section{Discussion}

The current study suggests that individuals in Europe currently treated for chronic urticaria have substantially worse outcomes than those without chronic urticaria across a variety of domains. This was true both in the comparisons matching for country, age, sex, and year of survey, as well as in regressions adjusting for BMI, smoking, and comorbidities within the matched samples. Differences in HRQOL exceeded commonly accepted 3-point minimally important differences for MCS and PCS scores; likewise, the 0.08-point decrement in SF-6D far exceeded the 0.03 threshold used for that measure [16, 22, 23]. Depression, anxiety, and sleep difficulties were also associated with current treatment for chronic urticaria. Health-related impairments in work and non-work activities and healthcare resource use were likewise greater among those with chronic urticaria than among controls.

It is important to consider that the cases in the current study reported current use of a prescription for chronic urticaria rather than CSU per se; the NHWS questionnaires in 2010-2013 did not include any items to differentiate between the types of chronic urticaria. Therefore, the burden quantified here may differ somewhat from that experienced by patients with confirmed diagnoses of CSU. However, as CSU is the most common type of chronic urticaria [5], many of the individuals with chronic urticaria in this cohort most likely have CSU, suggesting that this limitation is relatively minor. Though currently treated patients may be expected to have higher burden, which leads them to seek treatment, exploratory analyses comparing individuals who have either experienced chronic urticaria or been diagnosed with chronic urticaria also indicate substantial burden and are not limited to the proxy definition of the group reported upon here (ESM 2).

The findings should be considered in light of the limitations of the methodology. The survey is subject to potential biases arising from self-report, such as recall biases, but these biases would be expected to influence responses equally across analysis groups. Although the NHWS is representative of the included countries' adult populations in terms of age and sex, the panel-based recruitment may introduce potential bias for variables that are not incorporated in the sampling strata, such as education. Because both cases and controls were drawn from the same panel, this limitation would also be expected to have little impact on the estimated burden of the condition, though it could have affected the proportion of respondents with chronic urticaria. Likewise, because no description was provided for "chronic hives," some respondents experiencing the condition but unfamiliar with the term may not have been identified in the survey, which may have affected the prevalence estimate for undiagnosed patients. The SF-36v2, used in the NHWS to assess HRQOL, is a generic instrument that allows measurement of health status in any disease as well as in individuals free of disease. While this allows the health status of these patients to be compared with that of individuals without urticaria or other dermatological conditions, a more specific HR-QOL instrument would provide additional information and potentially a fuller description of the burden of the condition. As indicated earlier, all measures were self-report, which provides the patient perspective though is also potentially subject to bias. Measures also included different recall periods, ranging from 1 week for work and activity impairment to 1 year for anxiety, depression, and sleep 
difficulties. Healthcare use, which used a 6-month recall period, and psychological complaints, which were recalled over the prior 12 months, may not have coincided with treatment for chronic urticaria for some of the patients. Finally, though measured confounders were either used as matching variables or adjusted for in the regressions, there may have been unmeasured confounders that may account for some portion of the differences in health outcomes between cases and controls (e.g., family history of mental illness), and the cross-sectional design of the study does allow us to differentiate between the causes and consequences of chronic urticaria.

\section{Conclusions}

Despite the above limitations, the results of the current study using the proxy definition of chronic urticaria currently treated with a prescription suggest that CSU imposes a substantial burden on patient well-being and ability to be productive at work and in activities outside of work and is associated with more healthcare use. Depression, anxiety, and sleep difficulties were also notably more common among those currently treated for chronic urticaria. Research incorporating respondents with a confirmed diagnosis of CSU is needed to confirm these findings.

Acknowledgments MMB conceived the study, and JV conducted the analyses. All authors participated in interpreting the results, drafting the manuscript, and revising it for important intellectual content. All authors have read and approved the submitted version.

\section{Compliance with Ethical Standards}

This study was funded by Novartis and Genentech, which market a treatment for chronic urticaria (omalizumab). Editorial support was provided by Ghazaleh Samandari, PhD. Maria-Magdalena Balp is an employee of Novartis Pharma AG. Jeffrey Vietri and Gina Isherwood are employees of Kantar Health, to which Novartis paid fees for data access, analysis, and reporting. Haijun Tian is an employee of Novartis Pharmaceutical Corporation.

Open Access This article is distributed under the terms of the Creative Commons Attribution-NonCommercial 4.0 International License (http://creativecommons.org/licenses/by-nc/4.0/), which permits any noncommercial use, distribution, and reproduction in any medium, provided you give appropriate credit to the original author(s) and the source, provide a link to the Creative Commons license, and indicate if changes were made.

\section{References}

1. Zuberbier T, Aberer W, Asero R, Bindslev-Jensen C, Brzoza Z, Walter Canonica G, et al. The EAACI/GA2 LEN/EDF/WAO Guideline for the definition, classification, diagnosis, and management of urticaria: the 2013 revision and update. Allergy. 2013;2014(69):868-87.
2. Kaplan AP. Chronic urticaria: pathogenesis and treatment. J Allergy Clin Immunol. 2004;114(3):465-74.

3. Greaves M. Chronic urticaria. J Allergy Clin Immunol. 2000;105(4):664-72.

4. Maurer M, Weller K, Bindslev-Jensen C, Gimenez-Arnau A, Bousquet PJ, et al. Unmet clinical needs in chronic spontaneous urticaria. A GA2LEN task force report. Allergy. 2011;66:317-30.

5. Hellgren L. The prevalence of urticaria in the total population. Acta Allergol. 1972;27:236-40.

6. Gaig P, Olona M, Munoz Lejarazu D, Caballero M, Dominguez F, Echechipia S, et al. Epidemiology of urticaria in Spain. J Investig Allergol Clin Immunol. 2004;14:214-20.

7. Bakke P, Gulsvik A, Eide G. Hay fever, eczema and urticaria in southwest Norway: lifetime prevalences and association with sex, age, smoking habits, occupational airborne exposures and respiratory symptoms. Allergy. 1990;45:515-22.

8. Zuberbier T, Balke M, Worm M, Edenharter G, Maurer M. Epidemiology of urticaria: a representative cross-sectional population survey. Clin Exp Dermatol. 2010;35(8):869-73.

9. Weldon DR. Quality of life in patients with urticaria. Allergy Asthma Proc. 2006;27:96-9.

10. O'Donnell BF. Urticaria: impact on quality of life and economic cost. Immunol Allergy Clin N Am. 2014;34:89-104.

11. Kang MJ, Kim HS, Kim HO, Park YM. The impact of chronic idiopathic urticaria on quality of life in Korean patients. Ann Dermatol. 2009;21(3):226-29.

12. Staubach P, Eckhardt-Henn A, Dechene M, Vonend A, Metz M, Magerl M, et al. Quality of life in patients with chronic urticaria is differentially impaired and determined by psychiatric comorbidity. Br J Dermatol. 2006;154:294-8.

13. Bolge SC, Doan JF, Kannan H, Baran RW. Association of insomnia with quality of life, work productivity, and activity impairment. Qual life Res. 2009;18(4):415-22.

14. Liu GG, Dibonaventura MD, Yuan Y, et al. The burden of illness for patients with viral hepatitis $\mathrm{C}$ : evidence from a national survey in Japan. Value Health. 2012;15(1):S65-71.

15. Ware J, Kosinski M, Turner-Bowker DM, Gandek B. How to score version 2 of the SF-12 health survey. Lincoln: Quality Metric Incorporated; 2002.

16. Maruish ME, editor. User's manual for the SF-36v2 Health Survey, 3rd edn. Lincoln: Quality Metric Incorporated; 2011.

17. Brazier JE, Roberts J. The estimation of a preference-based measure of health from the SF-12. Med Care. 2004;42(9):851.

18. Reilly MC, Zbrozek AS, Dukes EM. The validity and reproducibility of a work productivity and activity impairment instrument. Pharmacoeconomics. 1993;4(5):353.

19. Charlson M, Szatrowski TP, Peterson J, Gold J. Validation of a combined comorbidity index. J Clin Epidemiol. 1994;47(11):1245-51.

20. Overton WS, Stehman SV. The Horvitz-Thompson theorem as a unifying perspective for probability sampling: with examples from natural resource sampling. Am Stat. 1995;49:261-8.

21. Current Population Survey, 2013 Annual Social and Economic (ASEC) Supplement [machine-readable data file]/conducted by the Bureau of the Census for the Bureau of Labor Statistics. Washington: US Census Bureau [producer and distributor]; 2013.

22. Norman GR, Sloan JA, Wyrwich KW. Interpretation of changes in health-related quality of life: the remarkable universality of half a standard deviation. Med Care. 2003;41:582.

23. Walters SJ, Brazier JE. Comparison of the minimally important difference for two health state utility measures: EQ-5D and SF6D. Qual Life Res. 2005;14:1523-32. 